\title{
Prospects for Observations of Microquasars with GLAST
}

\author{
Richard Dubois \\ for the LAT Collaboration \\ Stanford Linear Accelerator Center, Menlo Park CA, USA
}

\begin{abstract}
The Gamma-ray Large Area Space Telescope (GLAST) is a next generation high energy gamma-ray observatory due for launch in Fall 2007. The primary instrument is the Large Area Telescope (LAT), which will measure gamma-ray flux and spectra from $20 \mathrm{MeV}$ to $>300 \mathrm{GeV}$ and is a successor to the highly successful EGRET experiment on CGRO. The LAT will have better angular resolution, greater effective area, wider field of view and broader energy coverage than any previous experiment in this energy range. This poster will present performance estimates with particular emphasis on how these apply to studies of microquasars. The LAT's scanning mode will provide unprecedented unformity of sky coverage and permit measurements of light curves for any source. We will show results from recent detailed simulations that illustrate the potential of the LAT to observe microquasar variability and spectra, including source sensitivity and ability to detect orbital modulation.
\end{abstract}

PACS: 95.75.-z,95.75.Mn,95.75.Wx,95.80.+p,95.85.Pw

\section{INTRODUCTION}

The GLAST LAT is a high-energy pair conversion telescope that has been under development for over 10 years with support from NASA, DOE and international partners.

In normal operations the LAT will continually scan the sky, obtaining essentially complete sky coverage every 3 hours (two orbits). This uniformity of sky coverage together with the large effective area and good angular resolution should permit many advances in the study of microquasars in the GeV range. The most current LAT performance specifications are kept online[1].

\section{FEASIBILITY STUDY FOR MICROQUASAR OBSERVATIONS}

GLAST's Data Challenge 2 (DC2) provided a detailed simulation of the sky and the LAT's response. 5 X-ray binaries were included with flux and spectra from EGRET measurements and known orbital periods. Fifty-five days of simulated orbit were created using a full GEANT4 simulation of LAT response, with full time dependence in the simulations for AGN, solar flares, GRBs. Modeling of the XRB's was very simple, with single power law energy spectra and full modulation of the orbital dependence.

For initial studies into GLAST's capabilities in observing microquasars we originally concentrated on the two which have been measured in TeV gamma rays, by HESS[3] and MAGIC[4]. These provide good examples of the extremes of observations the LAT will be called on to make. The LAT's ability to determine the flux, spectral index and orbital time variability were discussed in this pilot study[2].

\section{Fast simulation of one year's orbit data}

A full year of orbit data was simulated using a parametrization of the instrument response and effective area in order to investigate how well we can see orbital modulation vs flux: Figure 1 shows LSI +61303 simulated with decreasing flux. This source is in a reasonably quiet part of the sky, and makes a best case example of the ability to see the orbital modulation. The LAT should be able to detect the modulation down to about a tenth of the nominal LSI +61 303 flux. We should be able to determine the sensitivity more quantitatively using periodograms.

Since most of the 'classic' microquasar candidates (eg GRS 1915+105, GX 399-4) are not steady, we have developed a new simulation model to examine the possible effects of the disk-jet cycle. The behaviour varies from random 


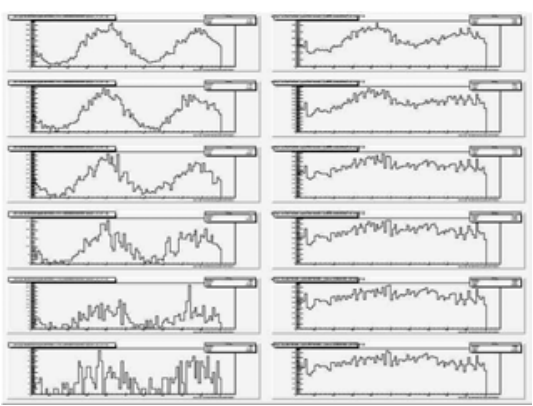

FIGURE 1. Orbital phase for LSI +61 303 vs flux. Nominal flux is at top, with each lower plot being a factor 2 lower in flux. The left column shows photons from LSI +61303 ; right shows all photons in a 2 degree radius.

outbursts to quasi periodic. The model accounts for a cycle in which the high energy jet is only on for a fraction of the time, allowing for fluctuations in the start time and duration of the jet-on time relative to the disk-jet cycle.

An initial test of the model was for GRS 1915+105, in which it was given a half-day disk-jet cycle, with the jet active for 3 hours of that time. The start time and duration was fluctuated by $10 \%$. Figure 2 shows the orbital phase vs flux and that the disk-jet cycle does not interfere with determining the orbital modulation.

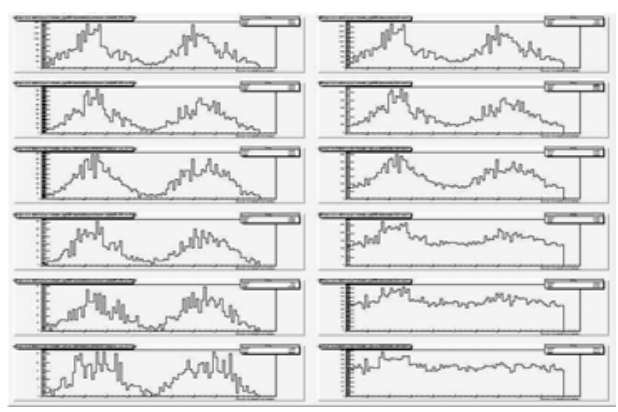

FIGURE 2. Orbital phase for GRS $1915+105$ vs flux. Nominal flux is at top, with each lower plot being a factor 2 lower in flux. The left column shows photons from LSI +61303 ; right shows all photons in a 2 degree radius.

\section{CONCLUSIONS}

For isolated sources, we will have no difficulty measuring the spectral and orbital period properties for sources with fluxes like those of LSI +61303 and LS $5039\left(\approx 10^{-7} \mathrm{ph} \mathrm{s}^{-1} \mathrm{~cm}^{-2}\right)$. For sources living in confused regions of the sky, like LS 5039, we will need to integrate longer and make harder energy cuts to bring the signal out above background. A better source localisation could in principle be achieved with a likelihood analysis that took into account that the PSF is a strong function of photon energy.

We have extended our simulations to examine non-steady sources, ranging from random outbursts to quasi periodic. The initial studies show that the disk cycle does not interfere with observing orbital modulation.

Prior to launch we plan to extend our full simulations to a full year of orbit data and develop the machinery to observe all the microquasar candidates. We will also investigate our ability to detect time variabilities other than orbital and develop strategies for multi-wavelength campaigns.

\section{REFERENCES}

1. S.Ritz et al, LAT Performance Specifications, http://www-glast.slac.stanford.edu/software/IS/glast_lat_performance.htm

2. R.Dubois Microquasar 6 Proceedings, Como, Italy Sept 2006

3. Aharonian et al, astro-ph 0607192

4. Alberts et al, Science, 2006 (1771) 312 